\title{
Genotying of Carbapenem Resistant Klebsiella pneumoniae from Clinical Isolates
}

\author{
M. Archana Hegde and A. Tejashree* \\ Department of Microbiology, JSS Medical College, Mysuru, India \\ *Corresponding author
}

\begin{tabular}{|l|}
\hline Ke y w or d s \\
Carbapenems, \\
Klebsiellapneumoni \\
ae, Blakpc gene, \\
Blandm-1 gene, \\
Blaoxa-48gene \\
\hline Article Info \\
\hline $\begin{array}{l}\text { Accepted: } \\
10 \text { April } 2019 \\
\text { Available Online: } \\
10 \text { May } 2019\end{array}$ \\
\hline
\end{tabular}

\section{A B S T R A C T}

$K$. pneumoniae is responsible for a wide variety of hospital and community-acquired infections, affecting patients with normal immune systems as well as those with pre-existing conditions. The Carbapenems are $\beta$-lactam antibiotics that are used in the treatment of infections caused by Extended Spectrum beta-Lactamases (ESBL) producing gram negative bacteria (GNB). Carbapenem antibiotics are considered the drugs of choice for the treatment of ESBL -producing Enterobacteriaceae and other multidrug resistant bacteria. However, resistance to carbapenems is being increasingly detected and is mainly related to the action of carbapenemase-type enzymes. The emergence of bacterial strains that produce carbapenemases further limits the therapeutic options available to clinicians. Genotying of carbapenem resistant Klebsiella pneumoniae from clinical isolates. This was a hospital based prospective study, undertaken in the department of Microbiology. All clinical isolates like Urine, Pus, ET secretions, BAL, Blood and other Body fluids received in the laboratory were subjected to routine processing as per standard procedures. Identification \&Antimicrobial susceptibility testing was performed by Vitek2 system. Modified Hodge test was carried out as confirmatory test. Genotypically, PCR was carried out for the detection of blaKPC, blaNDM-1 and blaOXA-48 gene. A total of 1,539 Klebsiella pneumoniae were isolated from various clinical samples over a period of 2 years from March 2015- April 2017. Among 1,539 Klebsiella pneumoniae isolates, 252(16.37\%) isolates were carbapenem resistant by Vitek 2.190(75.39\%) of the Carbapenem resistant Klebsiella pneumoniae were positive by Modified Hodge test. By PCR analysis blaKPC gene was found to be positive in $86(34.12 \%)$ of CRKP isolates. Followed by, blaNDM-1 gene showed $90(35.71 \%)$ positivity and $126(50 \%)$ isolates were positive for blaOXA-48 gene respectively. Carbapenem resistant is being progressively detected. This resistance is primarily related to the action of carbapenemase-type enzymes. The acceleratory frequency of carbapenemaseproducing bacteria indicates the urgency of having tools convenient to monitor the appearance and the spread of each family of carbapenemase gene types.PCR is a compelling method for detection of carbapenemase genes. This overcomes the limitations of the phenotypic tests. Hence molecular characterization should be considered.

\section{Introduction}

$K$. pneumoniae is responsible for a wide variety of hospital and community-acquired infections, affecting patients with normal immune systems as well as those with preexisting conditions K. pneumoniae is the most common cause of nosocomial respiratory tract and premature intensive care infections, and the most frequent cause of Gram-negative bacteraemia and urinary tract infections. Drug resistant isolates remain an important hospital-acquired bacterial pathogen, add significantly to hospital stays, and are 
especially problematic in high-impact medical areas such as intensive care units. This antimicrobial resistance is thought to be attributable mainly to multidrug efflux pumps. ${ }^{1}$ The ability of $K$. pneumoniae to colonize the hospital environment, including carpeting, sinks, flowers, and various surfaces, as well as the skin of patients and hospital staff, has been identified as a major factor in the spread of hospital-acquired infections. ${ }^{2}$ The Carbapenems are $\beta$-lactam antibiotics that are used in the treatment of infections caused by Extended Spectrum betaLactamases (ESBL) producing gram negative bacteria (GNB). Carbapenem antibiotics [Ertapenem, Imipenem, Meropenem, Doripenem, Razupenem (Faropenem)] are considered the drugs of choice for the treatment of Extended Spectrum Beta lactamase (ESBL)-producing Enterobacteriaceae and other multidrug resistant bacteria. ${ }^{3}$ However, resistance to carbapenems is being increasingly detected and is mainly related to the action of carbapenemas e-type enzymes. The emergence of bacterial strains that produce carbapenemases further limits the therapeutic options available to clinicians. Classes A to C have been well documented as both chromosomally encoded and plasmidmediated enzymes. ${ }^{4}$ The class D $\beta$-lactamases have been much more elusive and, for the most part, were identified only as plasmidencoded $\beta$-lactamases in Gram-negative bacteria.

The bacteria receiving the most attention is New Delhi metallo-beta-lactamase-1 (NDM1) producing superbug that confers resistance to most antibiotics including carbapenems. This carbapenemase is class B carbapenemase, also called metallo $\beta$ lactamases as they require zinc at their active site. This enzyme is coded by a gene called blaNDM-1. The NDM-1 enzyme was named after New Delhi, the capital city of India, as it was first described by Yong et al., in December 2009 when a Swedish national who fell ill with an antibiotic-resistant bacterial infection that he acquired in India. ${ }^{5}$ The infection was identified as a carbapenemresistant Klebsiella pneumoniae strain bearing the novel gene bla ${ }_{\mathrm{NDM}-1} .5$ Thereby organisms bearing blaNDM-1 gene have been identified from different parts of the world and it was considered that most of the cases were arisen from Indian subcontinent. ${ }^{6}$

Klebsiella pneumoniae Carbapenamases (KPC), identified in $2001^{7}$. The spread of antibiotic resistance genes such as NDM-1 and KPC is facilitated by horizontal gene transfer (HGT) between bacteria ${ }^{8}$. Among globally disseminated pathogens, HGT facilitates combination of the most effective antibiotic resistance genes from diverse geographies into multidrug resistance plasmids that spread between strains. Both HGT and clonal expansion have enabled KPC and NDM-1 to rapidly spread to distant locations after their emergence ${ }^{\text {? }}$.

In 2001, a Klebsiella pneumoniae isolate was obtained from a patient in Istanbul, Turkey, which was found to be multidrug resistant, including resistance to the carbapenems. In this isolate, a new OXA-type beta lactamase was identified and named OXA- $48^{10}$. This enzyme and its variants are now widespread in $K$. pneumoniae and other Enterobacteriaceae and have now been reported in A. baumanniias well ${ }^{11}$, and they represent one of the most concerning developments in carbapenem resistance in the last decade.

The best therapeutic approach to Klebsiella pneumoniae Carbapenemase producing organisms has yet to be defined; however, common treatments based on in-vitro susceptibility testing are the polymyxins, tigecycline, and less frequently 
aminoglycoside antibiotics are been used ${ }^{26}$. Hence, this study was undertaken to assess Genotying of carbapenem resistant Klebsiella pneumoniae from clinical isolates.

\section{Materials and Methods}

This was a hospital based prospective study, undertaken in the department of Microbiology, JSS Hospital. All clinical isolates of $K$. pneumoniae from patients attending out-patientand in-patient department at JSS hospital was included in the study. Fecal $K$. pneumoniae was excluded from this study. Inclusion criteria were phenotypically confirmed ESBL $K$. pneumoniae isolates for molecular detection of blaKPC, blaNDM-1 and blaOXA-48 gene. The samples collected were processed as per standard methods. The study protocol was approved by the ethics committee of the institute (Ref: JSS/MC/IEC/02/655/2015-16)

Urine, Pus, ET secretions, BAL, Blood and other Body fluids were received to the laboratory and were subjected to routine processing as per standard ${ }^{2}$ procedure by Vitek2 system.

\section{Modified Hodge test}

0.5 McFarland dilution of the E.coli ATCC 25922 was prepared in $5 \mathrm{ml}$ of broth or saline. $0.5 \mathrm{ml}$ of the $0.5 \mathrm{McFarland}$ was added to 4.5 $\mathrm{ml}$ of Mueller Hinton Broth (MHB) or saline to get 1:10 dilution. 1:10 dilution of E.coli ATCC 25922 was streaked as a lawn culture on to a Mueller Hinton agar plate and allowed to dry for 3-5 minutes. 10 $\mathrm{gg}$ Ertapenem susceptibility disc [CT1761B-ETP 10mcg, (B. No.-178667) Oxoid, UK.] was placed at the center of the test area. Test organism was streaked in a straight line from the edge of the disc to the edge of the plate. Up to four organisms can be tested on the same plate with one drug. Inoculated plates were incubated at $35^{\circ} \mathrm{C} \pm 2{ }^{\circ} \mathrm{C}$ in ambient air for 16-24 hours.

After 16-24 hours of incubation, the plates were examined for a cloverleaf-like indentation at the intersection of the test organism and the E. coli 25922, within the zone of inhibition of the Carbapenem susceptibility disc.MHT Positive test: Clover leaf-like indentation of E.coli 25922 growing along test organism growth streak within disk diffusion zone. MHT Negative test: No growth of E.coli 25922 along test organism growth streak within disc diffusion zone.

\section{blaKPC, blaNDM-1 and blaOXA-48Gene Detection}

DNA was extracted from overnight broth culture of $K$. pneumoniae, using HiPur A Bacterial Genomic DNA Purification Kit MB505 as per the manufacture's protocol. The blaKPC, blaNDM-1 and blaOXA-48 gene was identified by PCR using primers.

PCR tube consisted of $29 \mu 1$ of Master Mix + $1 \mu \mathrm{L}$ of DNA. The conditions included an initial denaturation step of $5 \mathrm{~min}$ at $95^{\circ} \mathrm{C}$, followed by 30 cycles of Holding Temperature: $95^{\circ} \mathrm{C}$, Annealing Temperature: $58^{\circ} \mathrm{C}$ and Extension for $45 \mathrm{sec}$ at $72^{\circ} \mathrm{C}$, Final extension step of $5 \mathrm{~min}$ at $72^{\circ} \mathrm{C}$. Isolates were screened for acquired blaKPC, blaNDM-1 and blaOXA-48 gene by PCR using primers and conditions described previously.

Post PCR Analysis by Gel Electrophoresis was carried out using 1\% Agarose (DNA grade, low melting, Himedia). The results were recorded on gel documentation system.

\section{Sequencing}

The PCR amplified product were purified using HiPurA PCR purification kit from HIMEDIA Labs Pvt. Ltd. The sequence 
chromatograms were analysed by BLAST and compared with known alleles to identify the replicons and identification of blaKPC, blaNDM-1 and blaOXA-48 alleles were done by using National Centre of Biotechnology Information database (www.ncbi.nlm.nih. gov/) (Table 1).

\section{Results and Discussion}

A total of 1,539 Klebsiella pneumoniae was isolated from various clinical samples (Bile, Blood, Urine, Sputum, drain, CSF, ET, CT, $\mathrm{PF}$, Pus) over a period of 2 years.

Out of 1,539 Klebsiella pneumoniae isolates, $252(16.37 \%)$ isolates were carbapenem resistant Klebsiella pneumoniae (CRKP) (resistant to Imipenem/ Ertapenem/ Meropenem antibiotics) by Vitek 2 .

\section{Modified Hodge Test (MHT) Result}

Out of 252 carbapenem resistant Klebsiella pneumoniae (CRKP) isolates, 190(75.39\%) were positive with clover-leaf indentation by Modified Hodge Test (MHT) and 62(24.60\%) were negative respectively. Chi square $=$ 65.016; $\mathrm{P}$ value $=0.000$ (very highly significant).

\section{Genotyping result}

The molecular detection of blaKPC gene, blaNDM-1 gene and blaOXA-48 genes was carried out for 252 CRKP isolates. $86(34.12 \%)$ isolates were positive for blaKPC gene, followed by $90(35.71 \%)$ were positive for blaNDM-1 gene and 126(50.0\%) were positive for blaOXA-48 gene by Polymerase chain reaction respectively with $\mathrm{P}$ value $=$ 0.000(Very Highly significant). The distribution of blaNDM-1, blaKPC and blaOXA-48 genes among CRKP isolates is shown in Table 2. Among 252 CRKP isolates $15(5.95 \%)$ isolates showed the presence of 3 genes (blaKPC+ blaNDM-1+ blaOXA-48).
Combination of KPC gene + NDM-1 gene showed 28(11.11\%) positive, followed by KPC gene + OXA-48 gene showed $38(15.07 \%)$ positive and NDM-1 gene + OXA-48 gene showed 43(17.06\%) positive with $\mathrm{P}$ value $=0.000$ (Very Highly significant) each respectively.

In the present study, an attempt was made to detect the Carbapenem Resistance in Klebsiella pneumoniae from various clinical samples by genotyping in a tertiary care hospital.

In our study, a total of 254(16.50\%) Klebsiella pneumoniae isolates were Carbapenem resistant from Vitek 2 result. Vijaya Doddaiah et al., ${ }^{12}$ (2014) showed $16.03 \%$ and Shalley Dahiya et al., ${ }^{13}$ (2015) showed the prevalence of $14.75 \%$ for CRKP.

Modified Hodge Test (MHT) is carried out to identify the existence of carbapenemase enzyme for isolates showing resistance to one/more carbapenems as advised by CDC. ${ }^{14}$. In the present study, 252 CRKP were tested for KPC and MBL production by Modified Hodge Test (MHT). Out of 252 carbapenem resistant Klebsiella pneumoniae (CRKP) isolates, 190(75.39\%) were positive with clover-leaf indentation for Modified Hodge Test (MHT) and 62(24.60\%) were negative respectively.

Our results are similar with many other studies like, $75 \%$ positive result showed by McGettigan et al., ${ }^{15}$ (2009) followed $69 \%$ by Amjad et al., ${ }^{16}$ Cury et al., (MHT 71\% positive) ${ }^{17}(2012)$ and little higher rate was seen in Galani et al., (2008) with $98 \%{ }^{18}$, and study by Shawn Vasoo et al., ${ }^{19}$ (2013) also showed $97.7 \%$ positive result respectively.

In the current study, out of 252 CRKP isolates, 86(34.12\%) isolates were positive for blaKPC gene by polymerase chain reaction. 
Similar to our study, Priyadarshini Shanmugam et al., ${ }^{20}$ in (2013) detected blaKPC gene, which showed $47.82 \%$ and another study by Efthymia Protonotariou et al. ${ }^{21}(2018)$ showed $45 \%$ positive.

Discordant results were observed in the study by Ana Carolina Ramos et al., ${ }^{22}$ (2018) detected $9.09 \%$ KPC gene, where as a study by Saritha Naya et al., ${ }^{23}$ (2014) showed $16.6 \%$ which was comparably low to our study. Sotgiu et al. ${ }^{24}$ (2018) observed 61\% positive for blaKPC gene followed by Aditya Raghunathan et al.,25 (2011) conducted a study that observed 96\% showing higher positive rates.

By PCR analysis, 90(35.71\%) isolates were positive for blaNDM-1 gene in our study. Identical to our study, Patrice Nordmann et al. ${ }^{26}$ (2012) investigated and reported that $37.5 \%$ of Carbapenem resistant Klebsiella pneumoniae were NDM-1 gene producers in 2012. Amin Abdelfattah Aqel et al., ${ }^{27}$ (2017) showed $50 \%$. In discordance with our study, Karthikeyan Kumaraswamy et al. ${ }^{28}$ (2010) investigated and reported that $9.9 \%$ of $K$. pneumoniae were NDM-1 producers from Chennai and in the same study $13 \%$ isolates showed positive for NDM-1gene from Haryana. A study conducted by Nagaraj et al., ${ }^{29}$ in 2012 showed that $75 \%$ isolates were positive for NDM-1gene. Another study by Arijit Bora et al., ${ }^{30}$ (2014) showed 71.79\% isolates were positive for NDM-1 gene.

In our study, occurrence of blaOXA-48 gene was found to be 126 (50\%) of CRKP K.pneumoniae. Identical to our study, Sara Lomonaco et al., ${ }^{31}$ (2018) observed 50\% blaOXA-48 gene type, followed by a study by Carole AyoubMoubareck et al., ${ }^{32}$ (2018) reported $53.3 \%$ positivity for blaOXA-48 gene. According to another study by Hamid Solgi et al., ${ }^{33}(2018)$ reported blaOXA-48 gene encoding in majority of CRKP isolates as $100 \%$. RymOuertani et al., ${ }^{34}$ (2018) and
Joseph D. Lutgring et al., ${ }^{35}$ (2018) also reported $92.85 \%$ and $90 \%$ positivity in their respective studies which was $40 \%$ higher when compared to our study. In discordance with our study, MarcArgente et al., ${ }^{36}(2018)$ reported $1.9 \%$, followed by Marianne Lund ${ }^{37}$ (2018)- 4.70\%, Sandra Pulss et al. ${ }^{38}$ (2018) $0.64 \%$, Martin Kaase et al., ${ }^{39}(2016)-5.8 \%$, these results were $50 \%$ lower when compared to the present study.

In the current study, 15(5.95\%) isolates showed the presence of 3 genes (blaKPC + blaNDM-1 + blaOXA-48). Combination of (blaKPC gene + blaNDM-1 gene) showed 28 $(11.11 \%)$ positive (P value 0.4 ), followed by (blaKPC gene + blaOXA-48 gene) showed $38(15.07 \%)$ positive (P value 0.1$)$ and (blaNDM-1 gene + blaOXA-48 gene) showed $43(17.06 \%)$ positive $(\mathrm{P}$ value $=0.5)$ respectively.

Similar to our study Sara Lomonaco et al., ${ }^{31}$ (2018) observed $20 \%$ positives in combination of (blaNDM-1 gene + blaOXA48 gene). Followed by Carole Ayoub Moubareck et al., ${ }^{32}$ (2018) identified both NDM-1 gene and blaOXA-48 gene with $22.1 \%$ positivity.

Amin Abdelfattah Aqel et al., ${ }^{40}$ (2018) identified $8.6 \%$ positive rate for both blaNDM-1 and baOXA-48 gene type which slightly lower to our study. Another study by Hamid Solgi et al., ${ }^{33}$ (2018) observed 87\% positives in combination of blaNDM-1 gene and blaOXA-48 gene showing higher positive result when compared to our study. The study conducted by Marianne Lund et al., ${ }^{41}$ (2018) and Fiona Senchyna et al., ${ }^{42}(2017)$ to detect blaKPC, blaNDM-1 and blaOXA-48genes showed correlating results.

Major part of the gut flora is subsidized by family Enterobacteriaceae where bla producing K.pneumoniae are also efficient to colonize. 
Table.1 Showing PCR primers used for KPC, NDM-1 and OXA-48 gene detection

\begin{tabular}{|c|c|c|c|}
\hline Gene & Primer & Oligonucleotide Sequence 5-3 & $\begin{array}{l}\text { Amplicon Size } \\
\text { (bp) }\end{array}$ \\
\hline 16SrDNA & $\begin{array}{l}\text { 16SrDNA_F } \\
\text { 16SrDNA_R }\end{array}$ & $\begin{array}{l}\text { F-5'-CCAGCAGCCGCGGTAATACG-3' } \\
\text { R-3'- } \\
\text { ATCGGTTACCTTGTTACGACTTC-5' }\end{array}$ & 996bp \\
\hline Class A: blaKPC gene & $\begin{array}{l}\mathrm{KPC} \_\mathrm{F} \\
\mathrm{KPC} \_\mathrm{R}\end{array}$ & $\begin{array}{l}F \text { - 5'-GTATCGCCGTCTAGTTCTGC-3' } \\
R \text { - 5'-GGTCGTGTTTCCCTTTAGCCA- } \\
3^{\prime}\end{array}$ & $635 b p$ \\
\hline $\begin{array}{l}\text { Class B: blaNDM-1 } \\
\text { gene }\end{array}$ & $\begin{array}{l}\text { NDM-1_F } \\
\text { NDM-1_R }\end{array}$ & $\begin{array}{l}\text { F- 5'-GGTTTGGCGATCTGGTTTTC-3' } \\
\text { R-5'-CGG AAT GGC TCA TCA CGA } \\
\text { TC-3' }\end{array}$ & $621 b p$ \\
\hline $\begin{array}{l}\text { Class D: blaOXA-48 } \\
\text { gene }\end{array}$ & $\begin{array}{l}\text { OXA-48_F } \\
\text { OXA-48_R }\end{array}$ & $\begin{array}{l}\text { F- 5'-TTGGTGGCATCGATTATCGG-3' } \\
\text { R- 5'-GAGCACTTCTTTTGTGATGGC- } \\
\text { 3' }\end{array}$ & 744bp \\
\hline
\end{tabular}

Table.2 Showing genotyping result

\begin{tabular}{|l|c|c|}
\hline \multicolumn{1}{|c|}{ Test } & Number & Percentage \\
\hline KPC gene Positive & 86 & $34.12 \%$ \\
\hline NDM-1 gene Positive & 90 & $35.71 \%$ \\
\hline OXA-48 gene Positive & 126 & $50.00 \%$ \\
\hline Presence all 3 genes(KPC+NDM-1+OXA-48) & 15 & $5.95 \%$ \\
\hline KPC gene + NDM-1 gene & 28 & $11.11 \%$ \\
\hline KPC gene + OXA-48 gene & 38 & $15.07 \%$ \\
\hline NDM-1 gene + OXA-48 gene & 43 & $17.06 \%$ \\
\hline
\end{tabular}

Image.1 Showing modified Hodge test result

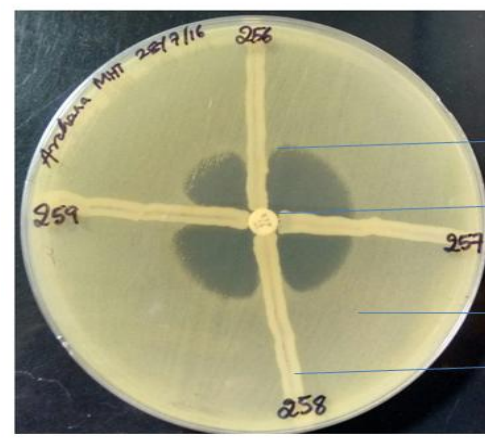

Positive result with

Cloverleaf indentation

Negative result without Cloverleaf indentation

ATCC 25922 E.coli lawn culture

Klebsiella pneumoniae test strain streak. 
Image. 2 Showing gel electrophoresis of KPC, NDM-1 and OXA-48 genes

Gel documentation of blaKPC, blaNDM-1 \& blaOXA-48 genes.

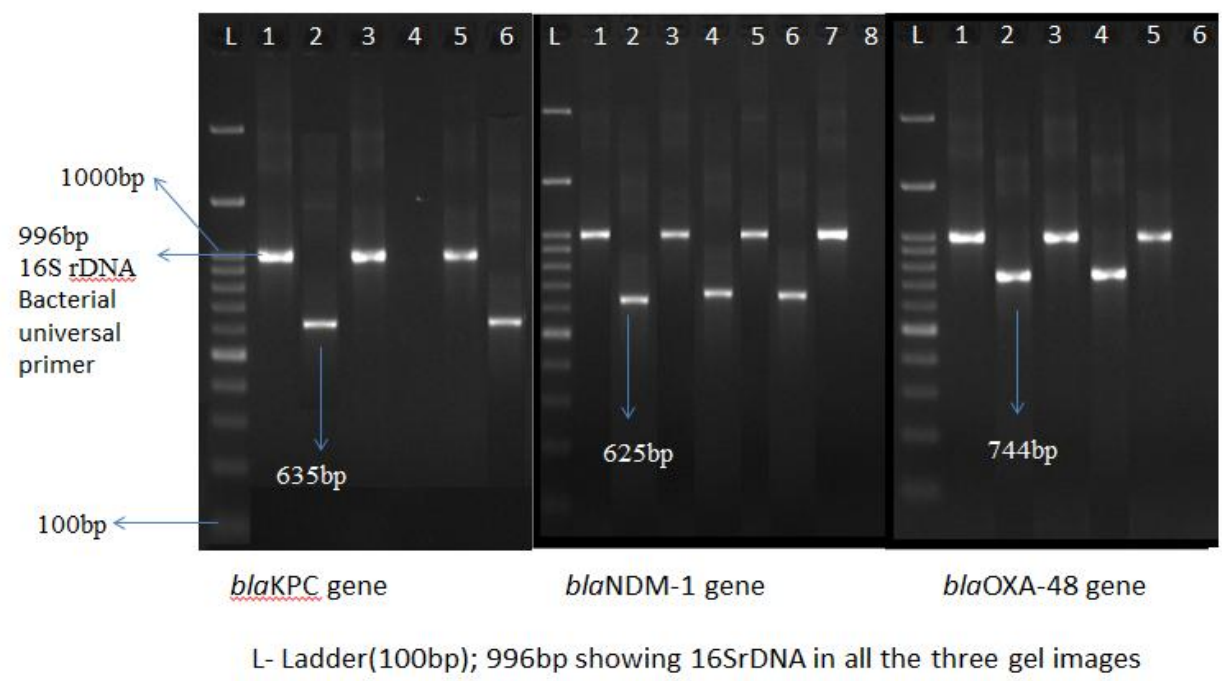

Based on these findings, genotypic assay could be considered in the diagnostic as confirmatory method for carbapenemase production and/or as an identification tool for the most important different carbapenemase genes.PCR is the rapid way to detect which family of $\beta$-lactamase is present when the presence of a carbapenemase is suspected. This finding is supported by Anjana Shenoy et al., ${ }^{43}$ (2014); Hrabak et al., ${ }^{44}$ (2014).

These in turn distribute as source for widening the infection especially in healthcare framework. In order to restraint the spread, disinfection procedures need to be implemented. Contact isolation of these infected patients is not routine. This may not be achievable in all healthcare settings, principally in the developing countries. Microbiological scrutiny at the time of admittance, contact isolation of potentially colonized patients, will go a rambling in hindering contamination of the environment and transmission to other patients. In addition, proper use of carbapenems will also hamper the selecting resistant bacteria in a habituated geographical area.

\section{References}

1. Cury, AP., Andreazzi D, Maffucci M, Caiaffa-Junior HH, Rossi F. The modified Hodge test is a useful tool for ruling out Klebsiella pneumoniae carbapenemase. Clinics 2012; 67 (12): 1427-31.

2. Ogawa, Wakano; Li, Dai-Wei; Yu, Ping; Begum, Anowara; Mizushima, Tohru; Kuroda, Teruo; Tsuchiya, Tomofusa. Multidrug resistance in Klebsiella pneumoniae MGH78578 and cloning of genes responsible for the resistance., Biological \& Pharmaceutical Bulletin. 2005, 28 (8): 1505-1508.

3. Datta, N., Kontomichalou P. Penicillinase synthesis controlled by infectious $\mathrm{R}$ Factors in Enterobacteriaceae. Nature 1965, 208: 239-244.

4. Ryan, S., Arnold, Kerri A. Thom, Saarika Sharma, Michael Phillips, et al., Emergence of Klebsiella pneumoniae Carbapenemase (KPC)Producing Bacteria. South Med J. 
2011; 104(1): 40-45.

5. Galani, I., Rekatsina DP, Hatzaki D, Plachouras D, Souli M, Giamarellou H. Evaluation of different laboratory tests for the detection of metallo- $\beta$ lactamase production in Enterobacteriaceae. J Antimicrob Chemother 2008; 61: 548-553.

6. Livermore, DM., Has the era of untreatable infections arrived? J Antimicrob Chemother, 2009, 64 Supp 1: i29-i36.

7. Nordmann P., Naas T, Poirel L. Global spread of carbapenemase producing Enterobacteriaceae. Emerg Infect Dis 2011; 17: 1791-8.

8. Yigit, H., Queenan AM, Anderson GJ, Domenech-Sanchez A, Biddle JW, Steward CD, et al. Novel carbapenemhydrolyzing beta-lactamase, KPC-1, from a carbapenem-resistant strain of Klebsiella pneumoniae. Antimicrob Agents Chemother. 2001; 45: 115161.

9. Hawkey, PM., Jones AM. The changing epidemiology of resistance. $\mathbf{J}$ Antimicrob Chemother. 2009; 64(Suppl 1): i3-10.

10. Hedges, RW., Datta N, Kontomichalou P, Smith JT. Molecular specificities of $\mathrm{R}$ factordetermined beta lactamases: correlation with plasmid compatibility. J. Bacteriol. 1974. 117: 56-62.

11. Poirel, L., Heritier C, Tolun V, Nordmann P., Emergence of oxacillinase-mediated resistance to imipenem in Klebsiella pneumoniae. Antimicrob. Agents Chemother. 2004. 48:15-22.

12. Vijaya Doddaiah, Dhanalakshmi Anjaneya. Prevalence of ESBL, AmpC and Carbapenemase among Gram Negative Bacilli Isolated from Clinical Specimens. American Journal of Life Sciences., 2014, 2, 2, pp.76-81.

13. Shalley Dahiya, PoojaSingla, Uma Chaudhary, Bijender Singh. Carbapenemases: A Review. International Journal of Advanced Health Sciences. 2015, Vol 2 Issue 4, Pp. 11-17.

14. Moran Gilad J, Carmeli Y, Schwartz D, Navon-Venezia S. Laboratory evaluation of the CHROM agar KPC medium for identification of carbapenem- nonsusceptible Enterobacteriaceae. Diagnostic Microbiology and Infectious Disease. 2011; 70(4): 565-567.

15. McGettigan, SE., Andreacchio K, Edelstein PH. Specificity of Ertapenem Susceptibility Screening for Detection of Klebsiella pneumoniae Carbapenemases. J Clin Microbiol 2009; 47: 785-786.

16. Amjad, A., Mirza I, Abbasi S, Farwa U, Malik N, Zia F. Modified Hodge test: A simple and effective test for detection of carbapenemase production. Iranian Journal of Microbiology. 2011; 3(4): 189-193.

17. Cury, AP., Andreazzi D, Maffucci M, Caiaffa-Junior HH, Rossi F. The modified Hodge test is a useful tool for ruling out Klebsiella pneumoniae carbapenemase. Clinics 2012; 67 (12): 1427-31.

18. Galani, I., Rekatsina DP, Hatzaki D, Plachouras D, Souli M, Giamarellou $H$. Evaluation of different laboratory tests for the detection of metallo- $\beta$ lactamase production in Enterobacteriaceae. J Antimicrob Chemother 2008; 61: 548-553.

19. Vasoo, S., Cunningham SA, Kohner PC, Simner PJ, Mandrekar JN, Lolans K, Hayden MK, Patel R. Comparison of a novel, rapid chromogenic biochemical assay, the Carba NP test, with the modified Hodge test for 
detection of carbapenemase-producing Gram-negative bacilli. J ClinMicrobiol. 2013. Sep; 51(9): 3097-101. doi: 10.1128/JCM.0096513.

20. Priyadarshini Shanmugam, Jeya Meenakshisundaram, Perumal Jayaraman, blaKPC gene Detection in Clinical Isolates of Carbapenem Resistant Enterobacteriaceaein a Tertiary Care Hospital, Journal of Clinical and Diagnostic Research. 2013, 7(12): 2736-2738.

21. Efthymia Protonotariou, AggelikiPoulou, LidaPoliti, Ioannis Sgouropoulos, et al., Hospital outbreak due to a Klebsiella pneumoniae ST147 clonal strain co producing KPC-2 and VIM-1 carbapenemases in a tertiary teaching hospital in Northern Greece., ijantimicag.2018.04.004.

22. Ana Carolina Ramos, Lygia Schandert, Cecilia G. Carvalhaes, Rafael Affini Martins, et al., Rapid detection of blaKPC directly from surveillance rectal swabs by EasyQ KPC., Diagnostic Microbiology and Infectious Disease 90. 2018., 251252.

23. Prasanth Manohar, Nazia Hassan, AbhaKumari, Vaikhari Kale, Nachimuthu Ramesh. Evaluation Of Phenotypic Methods Used For The Detection Of Carbapenemase: Modified Hodge Test, Carbapenem Inactivation Method, And Carbanp Test. Romanian Archives Of Microbiology And Immunology, 2018; 77, 2, Pp. 141-147.

24. G. Sotgiu, B.M. Are, L. Pesapane, A. Palmieri, N. Muresu, A. Cossu., Nosocomial transmission of carbapenem-resistant Klebsiella pneumoniae in an Italian university hospital: a molecular epidemiological study. Journal of Hospital Infection 99. 2018., 413-e418.

25. Aditya Raghunathan, Linoj Samuel and Robert J. Tibbetts., Evaluation of a Real-Time PCR Assay for the Detection of the Klebsiella pneumoniae Carbapenemase Genes in Microbiological Samples in Comparison With the Modified Hodge Test., Am J Clin Pathol 2011; 135: 566-571.

26. Nordmann, P., Boulanger AE, Poirel L. NDM-1 Metallo- $\beta$-Lactamase with Increased Carbapenemase Activity from Escherichia coli. Antimicrobial Agents and Chemotherapy. 2012; 56(4): 2184-2186.

27. Amin Abdelfattah Aqel, Jacqueline Findlay, Mohammad Al-Maayteh, Awatef Al-Kaabneh, Katie L. Hopkins, Hamed Alzoubi, Characterization of CarbapenemaseProducing Enterobacteriaceae from Patients in Amman, Jordan., mdr., 2017., 02, 38.

28. Kumarasamy KK, Toleman MA, Walsh TR, Bagaria J, et al., Emergence of a new antibiotic resistance mechanism in India, Pakistan and the UK: a molecular, biological and epidemiological study. Lancet Infect Dis, 2010; 10 (9): 597 602.

29. Nagaraj S, Chandran S P, Shamanna P, Macaden R. Carbapenem resistance among Escherichia coli and Klebsiella pneumoniae in a tertiary care hospital in south India. Indian J Med Microbiol 2012; 30:93-5

30. Bora A, Sanjana R, Jha BK, Narayan Mahaseth S, Pokharel K. Incidence of metallo-beta-lactamase producing clinical isolates of Escherichia coli and Klebsiella pneumoniae in central Nepal. BMC Research Notes. 2014; 7: 557. 
31. Sara Lomonac, Matthew A. Crawford, Christine Lascols, Ruth E. Timme, Kevin Anderson, David R. Hodge, Resistome of carbapenem- and colistin resistant Klebsiella pneumoniae clinical isolates PLoS ONE 13(6): e0198526.

32. Carole Ayoub Moubareck, Shaimaa F. Mouftah, TiborPál , Akela Ghazawi, Dalal H. Halat, AnjuNabi,. Clonal emergence of Klebsiella pneumoniae ST14 co-producing OXA-48-type and NDM carbapenemases with high rate of colistin resistance in Dubai, United Arab Emirates. International Journal of Antimicrobial Agents., 2018., 52 ., 90-95

33. Hamid Solgi. Molecular epidemiology of NDM-1- and OXA-48-producing Klebsiella pneumoniae in an Iranian hospital: clonal dissemination of ST11 and ST893 Journal of Antimicrobial Chemotherapy, 2018, 73, 6, Pg 15171524.

34. Ouertani R, Ben Jomàa-Jemili $M$, Gharsa H, Limelette A, Guillard T, Brasme L, de Champs C, Chouchani C. Prevalence of a New Variant OXA204 and OXA-48 carbapenemases plasmids encoded in Klebsiella pneumoniae clinical isolates in Tunisia. Microb Drug Resist. 2018 Mar; 24(2): 142-149. doi: 10.1089/mdr.2016.0236.

35. Joseph D. Lutgring, Wenming Zhu, Tom J.B. de Man, Johannetsy J. Avillan, Karen F. Anderson, David R. Lonsway, et al., Phenotypic and Genotypic Characterization of Enterobacteriaceae Producing Oxacillinase-48-Like

Carbapenemases, United States. Emerging Infectious Diseases, 2018; 24,4 .

36. Marc Argente, Elisenda Miró, CarminaMartí, AnaVilamala, Carles
Alonso-Tarrés, Frederic Ballester., Molecular characterization of OXA-48 carbapenemase producing Klebsiella pneumoniae strains after a carbapenem resistance increase in Catalonia. Enfermedades Infecciosas y Microbiología Clínica. J.eimc., 2018., 02.,003.

37. Marianne Lund, Marianne Bøgild Petersen, Anders Lægaard Jørgensen, Dorte Paulmann, Mikala Wang. Rapid real-time PCR for the detection of IMP, NDM, VIM, KPC and OXA-48 carbapenemase genes in isolates and spiked stool samples J. Diagmicrobio. 2018.04.002

38. Sandra Pulss, InkaStolle, IvonneStamm, Ursula Leidner, CarstenHeydel, Torsten Semmler, Ellen Prenger-Berninghoff and Christa Ewers. Multispecies and Clonal Dissemination of OXA-48 Carbapenemase in Enterobacteriaceae From Companion Animals in Germany, Front. Microbiol., 2016., 9:1265.

39. Martin Kaase, Sven Schimanski, Reinhold Schiller, Sören G Gatermann., Multicentre investigation of carbapenemase-producing Escherichia coli and Klebsiella pneumoniae in German hospitals. IJMM., 2016., 306(6).

40. Amin Abdelfattah Aqel, Jacqueline Findlay, Mohammad Al-Maayteh, Awatef Al-Kaabneh, Katie L. Hopkins, Hamed Alzoubi, Characterization of CarbapenemaseProducing Enterobacteriaceae from Patients in Amman, Jordan., mdr., 2017., 02, 38.

41. Marianne Lund, Marianne Bøgild Petersen, Anders Lægaard Jørgensen, Dorte Paulmann, Mikala Wang. Rapid real-time PCR for the detection of IMP, NDM, VIM, KPC and OXA-48 
carbapenemase genes in isolates and spiked stool samples. J. Diagmicrobio., 2018.04.002.

42. Senchyna F, Gaura RL, Sandlunda J, Truonga $\mathrm{C}$ et al. Diverse Mechanisms of Resistance in CarbapenemResistant Enterobacteriaceae at a Health Care System in Silicon Valley, California, bioRxlv. 2018 doi: http://dx.doi.org/10.1101/298513.

43. Shenoy KA, Jyothi EK, Ravikumar R. Phenotypic identification \& molecular detection of bla ndm-1 $_{1}$ gene in multidrug resistant Gram-negative bacilli in a tertiary care centre. The Indian Journal of Medical Research. 2014; 139(4): 625-631.

44. Hrabak J, Chudackova E, Papagiannitsis CC. Detection of carbapenemases in Enterobacteriaceae: a challenge for diagnostic microbiological laboratories. Clin Microbiol Infect 2014; 1469-0691.12678.

\section{How to cite this article:}

Archana Hegde, M. and Tejashree, A. 2019. Genotying of Carbapenem Resistant Klebsiella pneumoniae from Clinical Isolates. Int.J.Curr.Microbiol.App.Sci. 8(05): 825-835. doi: https://doi.org/10.20546/ijcmas.2019.805.098 